\title{
Changes in Synaptic Transmission and Long-term Potentiation Induction as a Possible Mechanism for Learning Disability in an Animal Model of Multiple Sclerosis
}

\author{
Ghasem Mosayebi ${ }^{1}$, Mohammad Reza Soleyman $^{1}$, Mostafa khalili ${ }^{1}$, Masoumeh Mosleh², Mohammad Reza Palizvan ${ }^{2}$ \\ ${ }^{1}$ Department of Microbiology and Immunology, Faculty of Medicine, Arak University of Medical Sciences, Arak, Iran \\ ${ }^{2}$ Department of Physiology, Faculty of Medicine, Arak University of Medical Sciences, Arak, Iran
}

Purpose: Multiple sclerosis (MS) is a chronic inflammatory demyelinating disease of the central nervous system. It has been shown that memory deficits is common in patients with MS. Recent studies using experimental autoimmune encephalomyelitis (EAE) as an animal model of MS have shown that indicated that EAE causes hippocampal-dependent impairment in learning and memory. Thus far, there have been no in vivo electrophysiological reports describing synaptic transmission in EAE animals. The aim of the present work is to evaluate the synaptic changes in the CA1 region of the hippocampus of EAE rats.

Methods: To evaluate changes in synaptic transmission in the CA1 region of the hippocampus of EAE rats, field excitatory postsynaptic potentials (fEPSPs) from the stratum radiatum of CA1 neurons, were recorded following Schaffer collateral stimulation.

Results: The results showed that EAE causes deficits in synaptic transmission and long-term potentiation (LTP) in the hippocampus. In addition, paired-pulse index with a $120 \mathrm{msec}$ interstimulus interval was decreased in the EAE group. These findings indicate that EAE might induce suppression in synaptic transmission and LTP by increasing the inhibitory effect of GAB$\mathrm{AB}$ receptors on the glutamate-mediated EPSP.

Conclusions: In conclusion, influence of inflammation-triggered mechanisms on synaptic transmission may explain the negative effect of EAE on learning abilities in rats.

Keywords: Encephalomyelitis, Autoimmune, Experimental; Hippocampus; Long-term Potentiation; Paired Pulse Index

- Fund Support: Financial support for this study was provided by Deputy Vice-chancellor of research on Arak University of Medical Sciences Grant \#1048.

- Research Ethics: Ethical approval for the study was provided by the Arak University of Medical Sciences Research Ethics Committee \# 92-159-11.

- Conflict of Interest: No potential conflict of interest relevant to this article was reported.

\section{INTRODUCTION}

Multiple sclerosis (MS) is a chronic inflammatory disease of the nervous system [1]. During this disease, lymphocytic infiltration into the central nervous system (CNS) results in the demy- elination and damage of axons. Between $40 \%$ and $60 \%$ of MS patients experience cognitive deficits such as learning and memory dysfunction [2]. Loss of cognitive function is one of the earliest manifestations of MS [3]. The mechanisms underlying these cognitive deficits remain poorly understood. It has
Corresponding author: Mohammad Reza Palizvan (iD http://orcid.org/0000-0001-7655-944X Department of Physiology, Faculty of Medicine, Arak University of Medical Sciences, Arak, IR, Iran

E-mail: dr.palizvan@arakmu.ac.ir /Tel: +98-861-4173502 / Fax: +98-861-4173529

Submitted: January 10, 2016 / Accepted after revision: March 1, 2016
This is an Open Access article distributed under the terms of the Creative Commons Attribution Non-Commercial License (http://creativecommons.org/licenses/by-nc/4.0/) which permits unrestricted non-commercial use, distribution, and reproduction in any medium, provided the original work is properly cited. 
been demonstrated that CNS inflammation could influence the induction of neuroplasticity and the function of neuronal networks, such as synaptic plasticity [4]. Postmortem hippocampal sections from MS patients show demyelination and neuropathology in the hippocampus [5]. Furthermore, hippocampal CA1 atrophy was reported in MS patients [6] and in an MS animal model [7].

Experimental autoimmune encephalomyelitis (EAE) is one of the best-studied animal models for MS. It is characterized by motor symptoms and cognitive impairments. Recently, Di Filippo et al. [8] reported that EAE could block the induction of long-term potentiation (LTP) in hippocampal slice preparations from mice. However, it is unclear whether EAE could effect in vivo LTP induction.

Thus, the aim of this study was to evaluate in vivo the effect of EAE on synaptic transmission, and LTP induced by the high frequency stimulation (HFS) of Schaffer collaterals in the CA1 region of the rat hippocampus. In addition, we used pairedpulse stimulation to determine whether the induction of EAE could alter inhibition or neurotransmitter release in the CA1 region of the hippocampal circuits.

\section{MATERIALS AND METHODS}

\section{Animals}

Forty male Wistar rats weighing 150 to $200 \mathrm{~g}$ were divided into 4 groups (2 control groups and 2 EAE groups) to measure synaptic transmission and LTP induction. The animals were housed under environmentally controlled conditions (12-hour light/dark cycles, temperature between $22^{\circ} \mathrm{C} \pm 2^{\circ} \mathrm{C}$ ) at the Arak University of Medical Sciences animal facility. Food and water were supplied ad libitum. All procedures were carried out in accordance with EU Directive 2010/63/EU and all regulations established by the Arak University of Medical Sciences Research Ethics Committee (\# 92-159-11).

\section{Induction of EAE}

EAE was induced by the subcutaneous injection of $100-\mu \mathrm{g}$ myelin basic protein (MBP) from guinea pig brain (Sigma Aldrich, St. Louis, MO, USA). The MBP was dissolved in $150 \mu \mathrm{L}$ of phosphate buffered saline and emulsified in an equal volume of complete Freund's adjuvant (Difco, BD, Franklin Lakes, NJ, USA) that included $0.2 \mathrm{mg}$ of Mycobacterium tuberculosis H37Ra (Difco). The anesthetized animals were injected on their hind limbs with MBP on day 0 and day 7. Two intraperitoneal injections (IP) of 200-ng pertussis toxin (Sigma Aldrich) were administered on day 0 and day 2 (postimmunization). Control animals were injected with vehicle solution. All animals were evaluated daily for clinical signs using the following clinical scale: 0 (no clinical signs), 1 (partial loss of tail tonus), 2 (tail paralysis), 3 (paraparesis of hindlimb), 4 (paraplegia), 5 (tetraparesis), 6 (tetraplegia), and 7 (death).

\section{Electrophysiology \\ Electrode positions}

Electrophysiology recording was initiated 28 hours after an animal obtained a score of at least 3 . Animals were anesthetized with urethane $(1.2 \mathrm{~g} / \mathrm{kg}, \mathrm{IP})$ and placed in the stereotaxic frame. Throughout the experiment, the rectal temperature was monitored and maintained at $36^{\circ} \mathrm{C}-37^{\circ} \mathrm{C}$ with a warming blanket. To stimulate the Schaffer collateral and commissural fibers, we used a twisted bipolar Teflon-coated stainless steel stimulating electrode (200- $\mu \mathrm{m}$ shaft diameter, A-M system, Carlsborg, WA, USA). We lowered the electrode vertically into the brain at 3.8 $\mathrm{mm}$ posterior and $2.8 \mathrm{~mm}$ lateral to bregma, through the dorsal hippocampus (depth, 2.8-3.4 mm). To record field potentials from the CA1 region of the hippocampus, we used a single Teflon-coated stainless steel electrode. The electrode was lowered vertically at $3.8 \mathrm{~mm}$ posterior and $2.0 \mathrm{~mm}$ lateral to bregma, through the dorsal hippocampus (depth, 2.8-3.4 mm). At the end of each experiment, the position of the electrodes was confirmed histologically.

\section{Stimulation and recording}

Electrophysiological (field potential) responses were recorded at the stratum pyramidale following stimulation of Schaffer collaterals. Electrical stimulation was supplied by a stimulus isolator unit (World Precision Instruments, Sarasota, FL, USA) and consisted of brief $(200 \mu \mathrm{sec})$ constant current square pulses. The average evoked field potentials were required to be stable for 60 minutes before tetanus delivery. When the variation in field population spike (PS) amplitude was less than $\pm 10 \%$ for 60 minutes, the baseline recording was considered stable. Animals that did not have stable baseline responses were excluded from the study. The response threshold was defined as the lowest stimulus intensity producing a consistently identifiable response. The stimulus intensity was then set to evoke a synaptic potential of a half-maximal PS. This stimulus intensity was also used for induction of LTP and to measure paired-pulse index (PPI). The PS amplitude was calculated as the average of the 
two potential differences of the negative spike peak to the preceding and following positive peaks.

For LTP induction, the tetanus consisted of 5 trains of $100 \mathrm{~Hz}$; each train lasting $200 \mathrm{msec}$ with an intertrain interval of $10 \mathrm{sec}-$ onds. After tetanus, the response was recorded for 60 minutes.

The PPI was established from the mean percent change in PS. The PPI was calculated as follows: (P2/P1) where P2 is the amplitude of the second PS and P1 is the amplitude of the first PS. The paired-pulse protocol averaged 5 responses with an interval of 60 seconds. The synaptic connections of the hippocampus produce two distinct phases of the PPI depending on the interstimulus interval (ISI), an early inhibitory phase (20-50 $\mathrm{msec})$, and a late facilitation phase (50-150 $\mathrm{msec}$ ) [9]. In our study, we chose $20 \mathrm{msec}$ ISI as the early phase and $120 \mathrm{msec}$ ISI as the late phase.

\section{Data Analysis}

We averaged 5 responses at each stimulus intensity level. The averaged waveform was used for further analysis. The PS amplitude $(\mathrm{mV})$ was determined from the averaged field potentials recorded from the stratum pyramidale. These signals were amplified (World Precision Instruments) and saved at a $10-\mathrm{kHz}$ sampling rate on a personal computer hard disk for off-line analysis (WSI, Tehran, Iran). We acquired the PS amplitude change over time mediated by HFS with respect to the 10 minutes baseline recording. The data was presented as mean \pm standard deviation. Unpaired or paired t-tests were used when two independent or de-

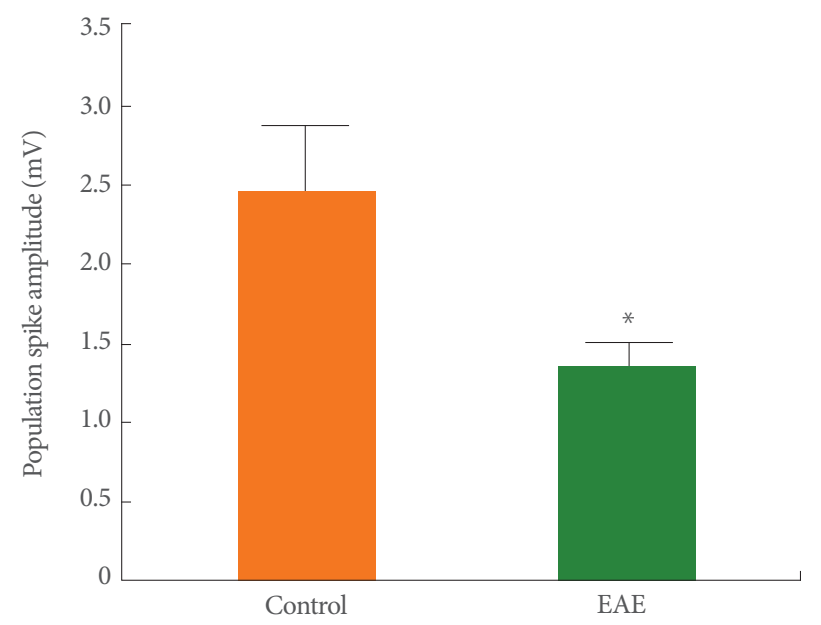

Fig. 1. Experimental autoimmune encephalomyelitis (EAE)mediated inhibition of CA1-synaptic transmission. The population spike amplitude in control $(\mathrm{n}=11)$ and $\operatorname{EAE}(\mathrm{n}=11)$ at low stimulus intensity. ${ }^{*} \mathrm{P}=0.042$, Unpaired t-test. pendent groups were compared, respectively. Differences were considered significant at $\mathrm{P}<0.05$.

\section{RESULTS}

\section{Synaptic Transmission}

Applying single pulses at $0.1 \mathrm{~Hz}$ to the Schaffer collateral inputs of CA1 neurons evoked a single PS in control and EAE rats. The CA1 field responses were increased in amplitude in proportion to the increase in stimulus intensity. The baseline responses for the control and EAE groups were stable at each stimulus intensity level with a stimulation rate of $0.1 \mathrm{~Hz}$. The mean values of the baseline PS amplitude for control and EAE groups are shown in Fig. 1. As shown in Fig. 1, the mean PS amplitude in EAE animals $(1.35 \mathrm{mV})$ are significantly lower than in the control rats $(2.46 \mathrm{mV})$ (Unpaired t-tests, $\mathrm{P}=0.042, \mathrm{n}=11$ ).

\section{Long-term Potentiation}

To understand the effect of EAE on synaptic plasticity, we analyzed LTP in the CA1 region. After recording stable field excitatory postsynaptic potentials (fEPSPs) from the CA1 region, a tetanic stimulation was applied to the Schaffer collaterals. Fig. 2 shows the time course change in PS amplitude. The amplitude of the PS in control rats increased to $142.5 \% \pm 15 \%$ of the baseline $(n=11)$ when measured 60 minutes after tetanic stimula-

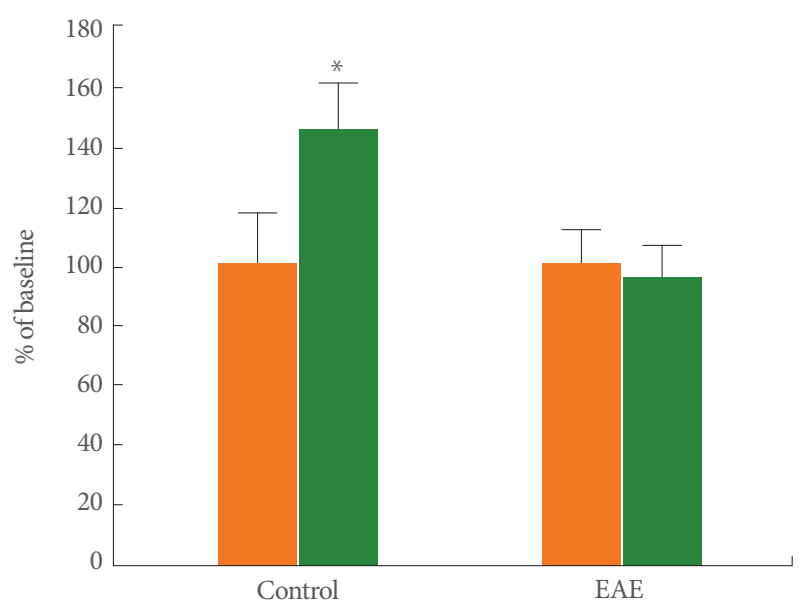

Fig. 2. Induction of population spike (PS) long-term potentiation (LTP) in control and experimental autoimmune encephalomyelitis (EAE) rats. The PS amplitude change vs. time in control $(\mathrm{n}=11)$ and $\operatorname{EAE}(\mathrm{n}=11)$ rats at the test stimulus intensity. Notice high frequency stimulation (HFS) induced PS LTP reversal 60 minutes after HFS in control but not in EAE rats. ${ }^{*} \mathrm{P}=0.001$, Paired t-test. 


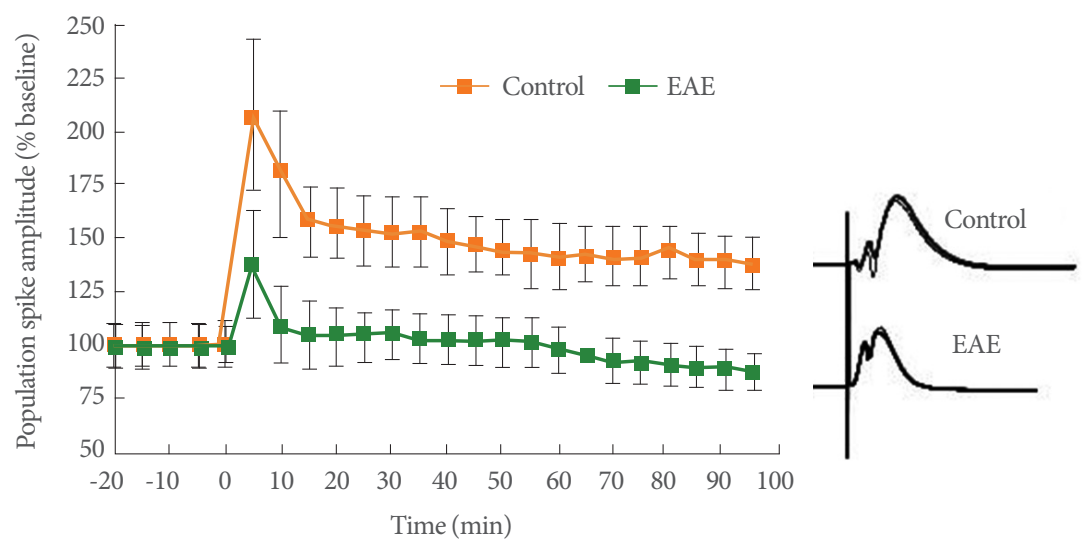

Fig. 3. Long-term potentiation is impaired in the Schaffer Collateral-CA1 synapses of the hippocampus in experimental autoimmune encephalomyelitis (EAE) rats, field population spike amplitude was plotted as percent of pretetanus baseline. Each point represents the mean \pm standard error of the mean. Right panel, representative averaged field potentials at the pyramidal cell layer in the control and EAE rats.
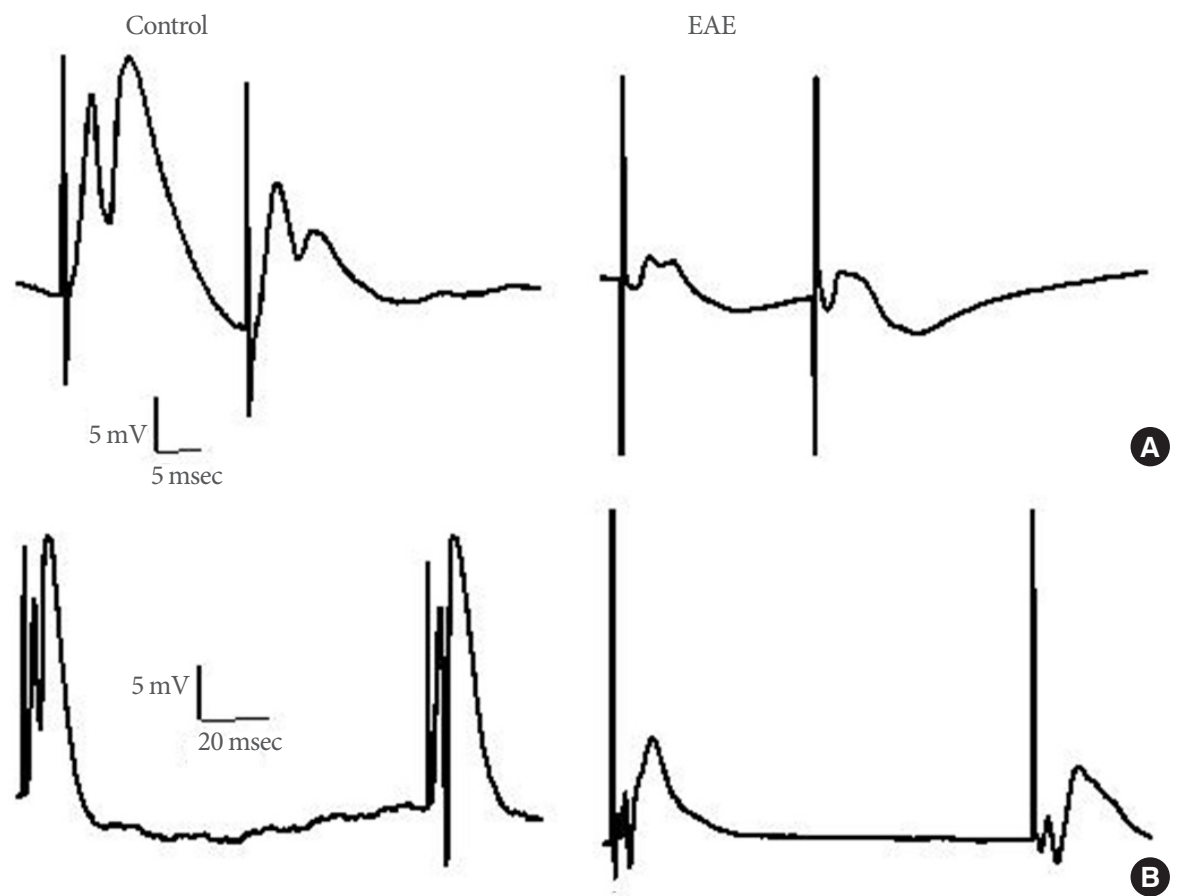

Fig. 4. Representative recordings of paired-pulse responses at the pyramidal cell layer in control and EAE rats. (A) Paired-pulse response at 20-msec, and (B) at 120-msec interpulse interval.

tion, which is indicative of LTP induction. Induction of EAE completely blocked the PS potentiation $(95.5 \% \pm 10.5 \%, \mathrm{n}=11)$, thus indicating that EAE could block LTP induction in the hippocampal CA1 region. Representative averaged field potentials at the pyramidal cell layer in control and EAE rats are shown in Fig. 3.

\section{Paired-Pulse Index}

To assess the impact of EAE on CA1 excitability at the circuit level, we determined the PPI of both animal groups using paired-pulse stimulation. Significant inhibition of the second population response was apparent at a $20 \mathrm{msec}$ interpulse interval in control $(\mathrm{n}=10)$ and in $\operatorname{EAE}(\mathrm{n}=8)$ rats. A comparison of the PPI in the 2 groups showed that with a $20 \mathrm{msec}$ interpulse 


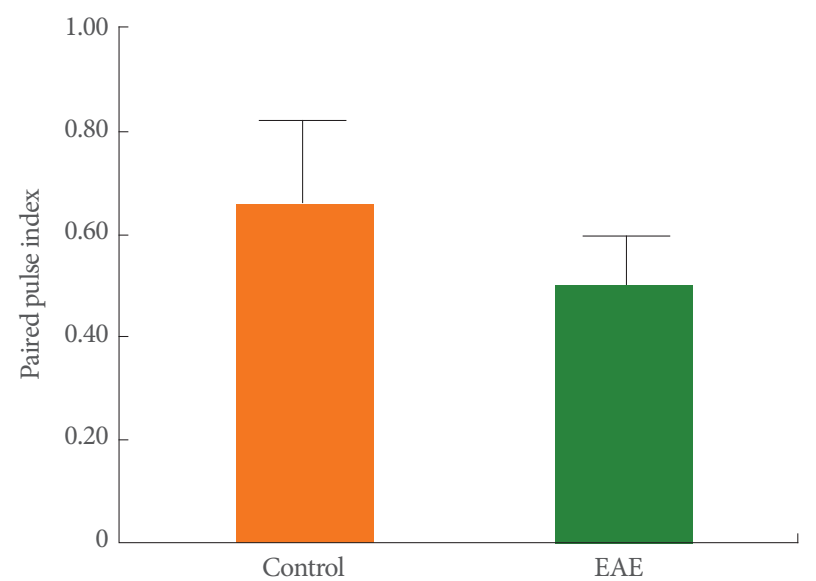

Fig. 5. Comparison of the paired-pulse index (PPI) in control $(n=10)$ and experimental autoimmune encephalomyelitis (EAE) $(n=8)$ affected rats at a 20 -msec paired-pulse interval. The PPI was decreased in EAE rats compared to control rats. Statistical analysis with Student t-test showed there was no significant difference between the 2 groups $(\mathrm{P}=0.455)$.

interval, the PPI was decreased in EAE rats as compared to control rats $(0.66 \pm 0.17$ in controls vs. $0.50 \pm 0.099$ in EAE rats). However, a Student t-test showed there was no significant difference between the 2 groups (Fig. 5). A paired-pulse paradigm with 120-msec ISI shows facilitation of the second population response. Statistical analysis of the PPI in control $(n=9)$ and in EAE $(n=6)$ rats with the Student t-test showed that PPI was significantly decreased in the EAE group as compared to control group ( $1.33 \pm 0.50$ in controls vs. $0.78 \pm 0.26$ in EAE rats, $\mathrm{P}=0.024$ ) (Fig. 6). Representative recordings of paired-pulse responses at 20- and 120-msec interpulse intervals in control and EAE rats are shown in Fig. 4.

\section{DISCUSSION}

The study's results indicate that activation of the peripheral immune system spread to the CNS and affected hippocampal neural transmission and neuroplasticity. Assessing spatial learning and memory in EAE rats is difficult since the disease can adversely affect motor performance. In this study, we used electrophysiology to study changes in hippocampal function during EAE for two reasons: (1) it is possible to assess hippocampal function without depending on animal locomotor activity, and (2) extensive synaptic changes in the hippocampus are believed to be responsible for deficits in hippocampal-dependent learning and memory. Although the effect of EAE on

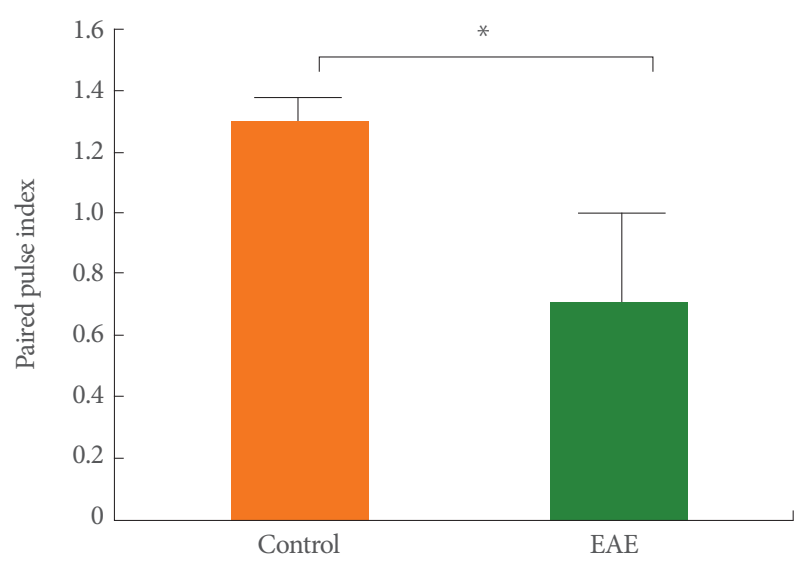

Fig. 6. Comparison of paired-pulse index (PPI) in control $(n=9)$ and experimental autoimmune encephalomyelitis (EAE) $(n=6)$ affected rats at a $120-\mathrm{msec}$ paired-pulse interval. The PPI was decreased in EAE rats compared to control rats. Statistical analysis with Student t-test showed a significant difference between the 2 groups. ${ }^{*} \mathrm{P}=0.024$.

hippocampal synaptic transmission has been previously report$\mathrm{ed}$, this is the first time that the effect of EAE on synaptic transmission and LTP was investigated in vivo.

Our results show that EAE caused a decrease in PS amplitude. Consistent with our results, an in vitro study on EAE showed that synaptic transmission was decreased in the CA1 region of the hippocampus [10]. In contrast, Di Filippo et al. reported that central and peripheral inflammation do not change basal synaptic transmission $[8,11]$. in contrast than the above situation where there was no change in transmission, Centonze et al. [12] found that EAE induction in mice increased striatal synaptic transmission.

We have observed that EAE inhibits LTP induction in the hippocampus. Since LTP represents a synaptic model of learning and memory, its impairment could be associated with deficits in spatial learning and memory, which could explain the high prevalence of MS-associated cognitive dysfunction. In support of this data, other studies have shown that long-term plasticity was decreased in EAE animals as compared to controls $[8,11]$. Recently, it was reported that anti-NMDA glutamate receptor antibodies are present in MS patients $[13,14]$. Therefore, the failure of HFS to induce LTP in the hippocampus could be explained by the presence of these autoantibodies. In contrast to our results, Nistico et al. [15] showed that in vitro, HFS could induce LTP in the hippocampal CA1 region. Moreover, the LTP levels were significantly enhanced in EAE slices as 
compared to controls [15]. The disparity in the data in regards to synaptic transmission and LTP induction may be attributed to different recording sites (CA1 region vs. striatum) or recording methods (in vitro vs. in vivo).

PPI is a phenomenon observed at many chemical synapses and reflects changes in neurotransmitter release [16]. Pairedpulse index were usually recorded with interpulse intervals between 20 and $300 \mathrm{msec}$. An inhibitory postsynaptic potential (IPSP) mediated by the GABAA receptor and $\mathrm{Cl}^{-}$, reaches its peak within 10-50 msec and lasts about $100 \mathrm{msec}$ [17]. As a result, we chose a 20-msec interpulse interval, which is selective for GABAA-mediated events [18]. Our results showed that EAE induction did not significantly alter PPI. Considering that a $\mathrm{K}^{+}$-mediated GABAB IPSP slowly reaches its peak within 13230 msec $[18,19]$, we used a 120 -msec interpulse interval to measure changes caused by EAE in GABAB-mediated events. We observed a significant reduction in the PPI of EAE animals as compared to controls, thus indicating that the efficacy of presynaptic GABAB receptors is increased in the hippocampal $\mathrm{CA} 1$ area of EAE rats. Presynaptic GABAB receptors are located at glutamatergic nerve terminals that regulate the release of glutamate from Schaffer collaterals to CA1 synapses [20,21]. Therefore, EAE might modulate suppression in synaptic transmission and synaptic plasticity by increasing the inhibitory effect of GABAB receptors on glutamate-mediated EPSPs.

In conclusion, the current study investigated the effect of EAE on synaptic transmission, LTP induction, and PPI in Wistar rats. The findings suggest that EAE might suppress synaptic transmission and LTP by increasing the inhibitory effect of GA$\mathrm{BAB}$ receptors in glutamate-mediated EPSP. This mechanism may explain the detrimental effect of EAE on the learning abilities of rats.

\section{REFERENCES}

1. Lassmann H, Bruck W, Lucchinetti C. Heterogeneity of multiple sclerosis pathogenesis: implications for diagnosis and therapy. Trends Mol Med 2001;7:115-21.

2. Calabrese P. Neuropsychology of multiple sclerosis: an overview. J Neurol 2006;253 Suppl 1:I10-5.

3. Rao SM, Leo GJ, Bernardin L, Unverzagt F. Cognitive dysfunction in multiple sclerosis. I. Frequency, patterns, and prediction. Neurology 1991;41:685-91.

4. Vereker E, O’Donnell E, Lynch MA. The inhibitory effect of interleukin-1beta on long-term potentiation is coupled with increased activity of stress-activated protein kinases. J Neurosci 2000;20:68119.

5. Papadopoulos D, Dukes S, Patel R, Nicholas R, Vora A, Reynolds R. Substantial archaeocortical atrophy and neuronal loss in multiple sclerosis. Brain Pathol 2009;19:238-53.

6. Sicotte NL, Kern KC, Giesser BS, Arshanapalli A, Schultz A, Montag $\mathrm{M}$, et al. Regional hippocampal atrophy in multiple sclerosis. Brain 2008;131(Pt 4):1134-41.

7. Ziehn MO, Avedisian AA, Tiwari-Woodruff S, Voskuhl RR. Hippocampal CA1 atrophy and synaptic loss during experimental autoimmune encephalomyelitis, EAE. Lab Invest 2010;90:774-86.

8. Di Filippo M, Chiasserini D, Gardoni F, Viviani B, Tozzi A, Giampà $\mathrm{C}$, et al. Effects of central and peripheral inflammation on hippocampal synaptic plasticity. Neurobiol Dis 2013;52:229-36.

9. Yorns WR Jr, Blaise JH, Bronzino JD. Frequency-dependent changes in the paired-pulse index in the hippocampus of the freely moving adult male rat. Exp Neurol 2004;186:104-8.

10. Ziehn MO, Avedisian AA, Dervin SM, Umeda EA, O’Dell TJ, Voskuhl RR. Therapeutic testosterone administration preserves excitatory synaptic transmission in the hippocampus during autoimmune demyelinating disease. J Neurosci 2012;32:12312-24.

11. Prochnow N, Gold R, Haghikia A. An electrophysiologic approach to quantify impaired synaptic transmission and plasticity in experimental autoimmune encephalomyelitis. J Neuroimmunol 2013;264: $48-53$.

12. Centonze D, Muzio L, Rossi S, Cavasinni F, De Chiara V, Bergami A, et al. Inflammation triggers synaptic alteration and degeneration in experimental autoimmune encephalomyelitis. J Neurosci 2009;29: 3442-52.

13. Uzawa A, Mori M, Takahashi Y, Ogawa Y, Uchiyama T, Kuwabara S. Anti-N-methyl D-aspartate-type glutamate receptor antibody-positive limbic encephalitis in a patient with multiple sclerosis. Clin Neurol Neurosurg 2012;114:402-4.

14. Pruss H, Dalmau J, Harms L, Holtje M, Ahnert-Hilger G, Borowski $\mathrm{K}$, et al. Retrospective analysis of NMDA receptor antibodies in encephalitis of unknown origin. Neurology 2010;75:1735-9.

15. Nistico R, Mori F, Feligioni M, Nicoletti F, Centonze D. Synaptic plasticity in multiple sclerosis and in experimental autoimmune encephalomyelitis. Philos Trans R Soc Lond B Biol Sci 2013;369:20130162.

16. Chu Z, Hablitz JJ. GABA(B) receptor-mediated heterosynaptic depression of excitatory synaptic transmission in rat frontal neocortex. Brain Res 2003;959:39-49.

17. Karnup S, Stelzer A. Temporal overlap of excitatory and inhibitory afferent input in guinea-pig CA1 pyramidal cells. J Physiol 1999; 516(Pt 2):485-504. 
18. Davies CH, Davies SN, Collingridge GL. Paired-pulse depression of monosynaptic GABA-mediated inhibitory postsynaptic responses in rat hippocampus. J Physiol 1990;424:513-31.

19. Zeng X, Tietz EI. Depression of early and late monosynaptic inhibitory postsynaptic potentials in hippocampal CA1 neurons following prolonged benzodiazepine administration: role of a reduction in Cl- driving force. Synapse 1997;25:125-36.
20. Lanthorn TH, Cotman CW. Baclofen selectively inhibits excitatory synaptic transmission in the hippocampus. Brain Res 1981;225:1718.

21. Potier B, Dutar P. Presynaptic inhibitory effect of baclofen on hippocampal inhibitory synaptic transmission involves a pertussis toxin-sensitive G-protein. Eur J Pharmacol 1993;231:427-33. 